\title{
A Review Paper on Multimodal Biometrics System using Fingerprint and Signature
}

\author{
Sangramsing $\mathrm{N}$. \\ Kayte \\ Research Scholar \\ Department of \\ Computer Science \& \\ IT, \\ Dr. Babasaheb \\ Ambedkar \\ Marathwada \\ University, \\ Aurangabad, India
}

\author{
Suvarnsing G. \\ Bhable \\ Research Scholar \\ Department of \\ Computer Science \& \\ IT, \\ Dr. Babasaheb \\ Ambedkar \\ Marathwada \\ University, \\ Aurangabad, India
}

\author{
Jaypalsing N. \\ Kayte \\ Research Scholar \\ Department of \\ Computer Science \& \\ IT \\ , Dr. Babasaheb \\ Ambedkar \\ Marathwada \\ University, \\ Aurangabad, India
}

\author{
Raju Maher \\ Research Scholar \\ Department of \\ Computer Science \& \\ IT, \\ Dr. Babasaheb \\ Ambedkar \\ Marathwada \\ University, \\ Aurangabad, India
}

\begin{abstract}
A biometric system is important to a pattern recognition system that operates by acquiring biometric data from an individual, extracting a feature set from the acquired data, and comparing this feature set against the template set in the database. Multimodal biometric systems are becoming more popular; Fingerprint recognition is the most popular physiological characteristic used to identify a person in biometric systems, because of feasibility, permanence, distinctiveness, reliability, accuracy, and acceptability Signature recognition is the most popular behavioral characteristic used in biometric systems. Thus, we believe that the combination of these two methods will have a reliable and accurate result. We propose a weighted fusion scheme, which transforms the scores into a common range, assigned weights and combines them, giving the final fused score.
\end{abstract}

\section{Keywords}

Fingerprint, Signature, fusion image, ICP algorithm, Euclidean distance Fingerprint, Signature, fusion image, ICP algorithm, Euclidean distance.

\section{INTRODUCTION}

The fingerprint based system provide high level of uniqueness, the compact solid state fingerprint sensors can be easily embedded into wide variety of devices which are used for user authentication. But these solid state sensors can sense only a limited portion of fingerprints and therefore, the amount information obtained is also limited. So in order to address this problem of limited information in a single fingerprint template, mosaicking technique is used. What is Mosaicking Techniques? The multiple impression of same fingerprint are fused together of resulting in a more complete fingerprint templates. So this fused image is called as composite image. Following are the advantages of composite template [1] -

1. Instead of comparing the query image which each of the individual images of same finger a composite image enables the reduction of number of comparisons(only one comparisons)and their by reducing the probability of false reject.

2. b] It reduces the matching time since only one comparison is made. The signature verification and recognition are used in banking transactions, electronic funds transfer, and document analysis.
Signature verification can be of two types-offline and online system. The online system consists of a pen and epad. The offline system consists of images that are previously

stored in the database and then after processing these images are compared and verified. However the offline signature are more complex than the online signatureverifications. There are several factors that affect offlineSystem such as type of pen used for the signature, the fancy handwriting style and the non repetitive nature of the variations of the signature.

\section{FINGERPRINT}

While registering any fingerprint image due to the Non Uniform pressure applied by the subject, the fingerprint image may have non linear plastic distortions. A noisy image may be formed due to the presence of dirt on the sensor or bruises on finger. So if two images of the same finger have different amount of noise or distortion then it is quite difficult to register them. This can be reduced by using registration algorithm, which finds transformation $\mathrm{T}$ such that it relates the two images of the same finger. Suppose $\mathrm{X}$ and $\mathrm{Y}$ are the two entities and Rx and Ry their range image respectively. The intensity values are directly used as range values - i.e., the intensity value of the image at the planar coordinate $(x, y)$ is treated as the range value, $\mathrm{z}$, at that location. The goal of a registration algorithm is to find $\mathrm{T}$ such that the objective function,

$\mathrm{D}(\mathrm{Rx}, \mathrm{Ry})$, is minimized:

$\mathrm{D}(\mathrm{Rx}, \mathrm{Ry})=\Sigma\|\mathrm{Tx}-\mathrm{f}(\mathrm{x})\|$

Where $\mathrm{f}: \mathrm{Rx} \rightarrow \mathrm{Ry} \mid ¥ \mathrm{x} \in \mathrm{Rp}, \mathrm{f}(\mathrm{p}) \in \mathrm{Ry}$.

We express transformation matrix, $\mathrm{T}$, in homogeneouscoordinates. Equation(2) shows the transformation matrix where $\alpha, \beta$ and $\gamma$ are the rotation angles about $\mathrm{x}, \mathrm{y}$ and $\mathrm{z}$ axes respectively, $\mathrm{tx}, \mathrm{ty}, \mathrm{tz}$ are the translation components along the three axes. In practice since the $\mathrm{f}$ is not known, so the objective function in eq (1) is replaced by the evaluation function that covers in itself the information of a set of corresponding points in Rx and Ry.consider $\mathrm{N}$ pairs of corresponding points, (pi,qi), pi $€ \mathrm{Rx}$, qi $€ \mathrm{Ry}$ and $\mathrm{i}=1,2, \ldots \ldots \ldots \mathrm{N}$.

The evaluation function $\mathrm{E}(\mathrm{Rx}, \mathrm{Ry})$ is given by:

$\mathrm{E}(\mathrm{Rx}, \mathrm{Ry})=\Sigma \|$ Txi - qi $\| 2 \ldots \ldots . .(3)$ 
The high level features like corners, edges, etc. are extracted from the two surfaces, for selecting the correspondence points. The correspondence points are also called as control points. In some application the correspondence points are manually identified by the expert domain. So given the correspondence points, the evaluation function $\mathrm{E}(\mathrm{Rx}, \mathrm{Ry})$ in eq (3) can be minimized by searching for the global minimum in the 6 dimensional parameter space using iterative procedure, though it does not guarantee convergence to global minimum. So to solve this problem, the iterative closest point(ICP) algorithm assumes that initial approximate T0 is already known. If the approximation of this T0 is good then the global minimum can be reached quickly and surely. The

ICP algorithm tries to minimize the distances between points in one image to geometric entities in the other.[2]

We minimize it using:

$\operatorname{Ek}(\mathrm{Rp}, \mathrm{Rq})=\Sigma(\mathrm{ds}) 2(\mathrm{Tkpi}, \mathrm{Sjk})$

$\mathrm{Ds}=$ distance form point to the plane

$\mathrm{Sj}=$ tangential plane corresponding to point qj in image $\mathrm{Ry}$ After the initial alignment by examining homogeneous regions in the two images, the control points are automatically chosen. ICP is used to minimize the function. Since an approximate initial transformation matrix is known the convergence is faster.

\subsection{Fingerprint Mosaicking}

Please By using the modified ICP algorithm, the problem of 3D surface registration can be solved.

What is minutiae point? The terminations or the bifurcations formed by the ridges present in fingerprint image are called as minutiae points.

The minutiae point extraction is performed on each individual image. For each image we have different set of minutiae points. The two sets of the minutiae points are compared by using elastic point matching algorithm. The reference minutiae pair is selected such that one minutiae point is from one image and another point is from the other image. The number of corresponding minutiae pairs is determined using the remaining set of the points. A pair is considered as a reference pair, if it gives large number of corresponding pairs.

Consider (pi,qi)........(pN,qN) be the corresponding minutiae pairs

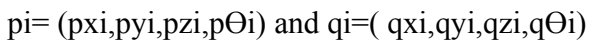

$(\mathrm{x}, \mathrm{y})=$ spatial coordinates of minutiae points

$\mathrm{Z}=$ intensity of image at $(\mathrm{x}, \mathrm{y})$

$\Theta=$ minutiae orientation

$\mathrm{T}=$ Transformation matrix

The initial transformation T0 is calculated using Hornes method [5], that operates on the $(\mathrm{x}, \mathrm{y}, \mathrm{z})$ values. The translation parameters viztx ,ty, tzare computed using the centroid of the point sets (pxi ,pyi , pzi ) and

(qxi , qyi , qzi ), and the rotation components are computed using the cross covariance matrix between the centroidadjusted pairs of points[2].

\subsection{Construction of a Composite}

By using the final transformation matrix i.e. Tsolution, the two intensity images Ip and Iqare integrated to form a new image Ir, to compute the new spatial coordinate of every pixel in Ip. the new minutiae set is then extracted from Ir. The composite image is then subjected to the preprocessing techniques, minutiae extraction and post processing stage as explained further.

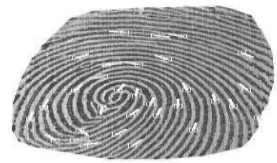

(a)Minutiae extracted

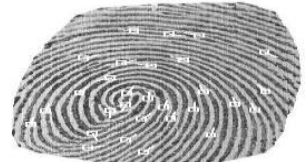

(b) Composite minutiae set
This composite image is then set to match with the fingerprint image which we obtain dynamically through the sensor. The fingerprint image obtained dynamically undergoes the following processing

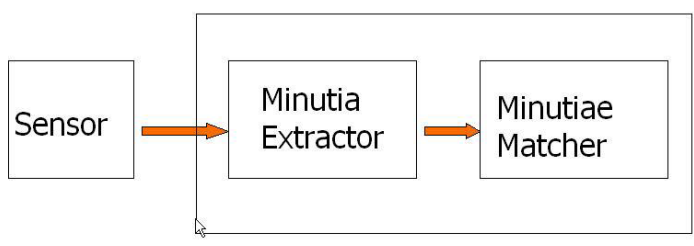

Fig 1: System for Fingerprint Recognition

\subsection{Algorithm Level Design For Matching}

The composite image and the image obtained dynamically are subjected to matching by using the following approach. Both the images undergo through the stages individually, and later are set for minutiae matching. The three stage approach to implement minutiae extractor is:

- Pre-processing,

- Minutia extraction

- $\quad$ Post-processing stage.

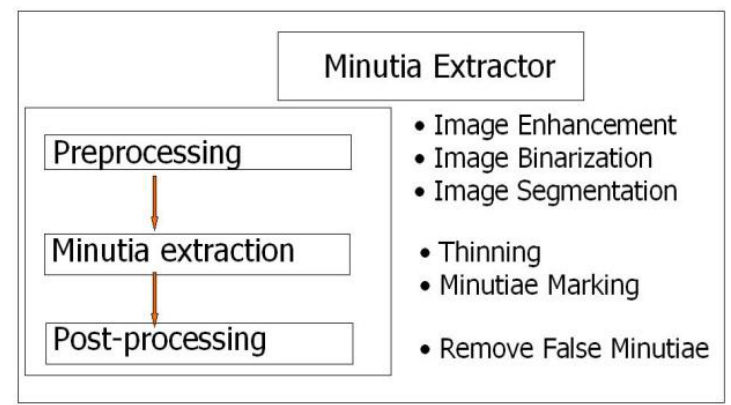

Fig 2: Minutia Extractor

\subsection{Fingerprint Image Preprocessing}

In order to make further operations clearer it is mandatory to make the image clearer. The images acquired from the sensors or other Medias are not of perfect quality, so therefore we need to apply some processing to make the image clearer and usable for the operations in order to perform matching. The following are the pre-processing operations applied on an image:

\subsubsection{Image Enhancement:}

The process of enhancement increases the pixel value of an image. The enhancement can be carried out by two methods:

i) Histogram equalization

ii) Fast Fourier transformation 
1. Image enhancement by Histogram equalization: It increases the perceptional information by expanding the pixel value distribution of an image[3]. The original

histogram of a fingerprint image has the bimodal type (fig. 4), the visualization effect is enhanced and the histogram after the histogram equalization occupies all the range from 0 to 255 (fig 5) .
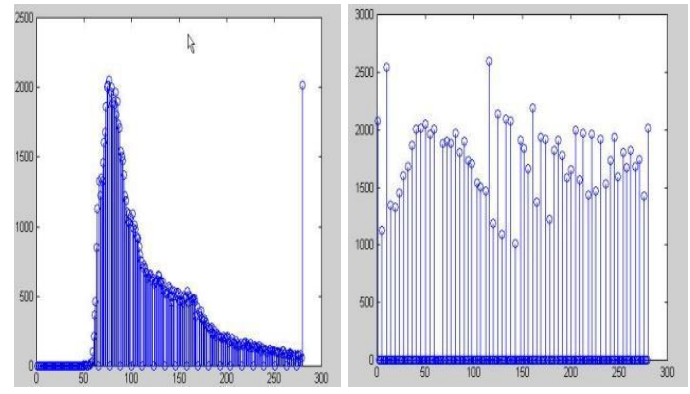

Fig 3. Original histogramof Fingerprint image

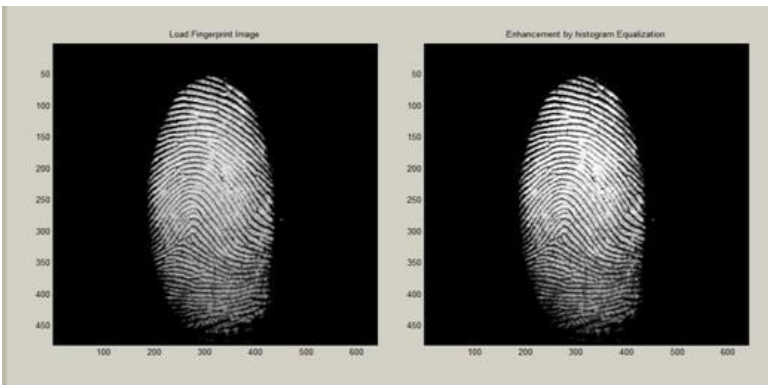

Fig 5. Image enhancement by histogram equalization

\section{5(a).Original image 5(b).Image after histogram} equalization

\section{Fingerprint Enhancement by Fourier Transform:}

The image is divided into small processing blocks of 32 by 32 pixels. The Fourier transform is performed according to:

$$
F(u, v)=\frac{1}{N M} \sum_{x=0}^{N} \sum_{y=0}^{M} f(x, y) e^{-j 2 \pi\left(\frac{u x}{N}+\frac{v y}{M}\right)}
$$

For $\mathrm{u}=0,1,2,31$ and $\mathrm{v}=0,1,2,31$.

In order to enhance a specific block by its dominant frequencies, we multiply the FFT of the block by its magnitude for a specific set of times. Where the magnitude of the original

$$
\mathrm{FFT}=\operatorname{abs}(\mathrm{F}(\mathrm{u}, \mathrm{v}))=|\mathrm{F}(\mathrm{u}, \mathrm{v})| .
$$

Enhanced block is obtained according to: $\mathrm{g}(\mathrm{x}, \mathrm{y})=\mathrm{FF}(\mathrm{u}, \mathrm{v}) \mathrm{x}$ $\mathrm{F}(\mathrm{u}, \mathrm{v}) \mid$ ..(2),

Where $\mathrm{F}-1(\mathrm{~F}(\mathrm{u}, \mathrm{v}))$ is obtained by:

$$
f(x, y)=\frac{1}{M N} \sum_{x=0}^{M-1} \sum_{y=0}^{N-1} F(u, v) \times \exp \left\{j 2 \pi \times\left(\frac{u \pi}{M}+\frac{v y}{N}\right)\right\}(3)
$$

for $\mathrm{x}=0,1,2, \ldots, 31$ and $\mathrm{y}=0,1,2, \ldots, 31$.

The value of $\mathrm{k}$ in formula (2) is determined experimentally and is called as experimentally determined constant. We generally choose $k=0.45$ to calculate. Higher the value of "k" more is the improvement in the appearance of the ridges and can fill up small holes in ridges, having too high "k" can result in false joining of ridges resulting in termination becoming a bifurcation. Fig 7.presents the image after FFT enhancement.

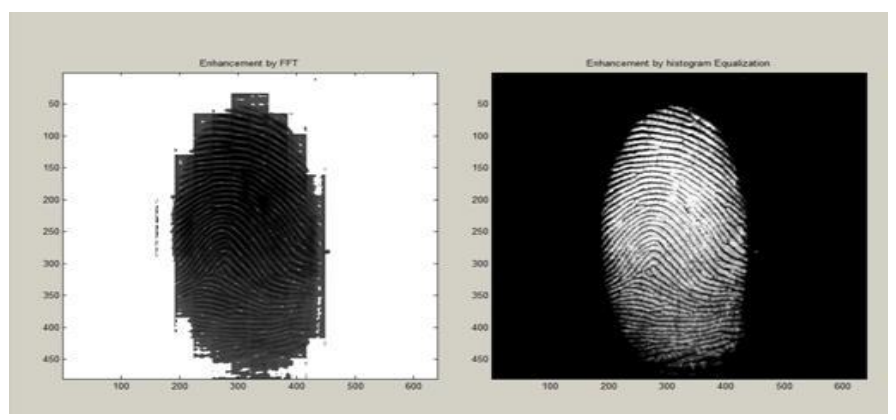

Fig 6.Fingerprint enhancement by FFT Enhanced image (left), Original image (right)

Here it can be seen that the enhanced image after FFT has the improvements to connect some falsely broken points on ridges and to remove some spurious connections between ridges. The shown image at the left side of figure is also processed with histogram equalization after the FFT transform.

\subsubsection{Fingerprint Image Binarization}

The 8 bit gray fingerprint image is transformed to a 1 bit image where 0 -value is for ridges and 1 -value is for furrows. Ridges in the fingerprint are highlighted with black color while furrows are white after this pre-processing. To binarize the fingerprint image we perform locally adaptive binarization method. It transforms a pixel value to 1 if the value is larger than the mean intensity value of the current block (16x16) to which the pixel belongs [Fig 7].

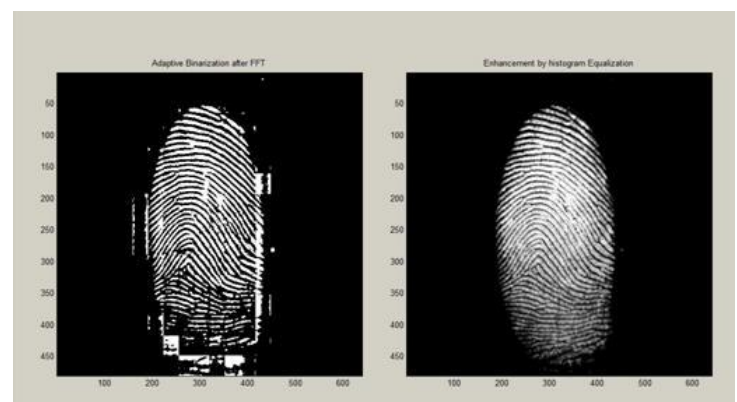

Fig 7. Fingerprint image after adaptive binarizationBinarizedimage(left), Enhanced gray image(right)

\subsubsection{Fingerprint Image Segmentation}

The image area that holds background information that is

without effective ridges and furrows is first discarded. Then the bound of the remaining effective area is sketched out, since the minutia in the bound region is confusing with that spurious minutia that is generated when the ridges are out of the sensor. This is called as region of interest (ROI). The 2 step method is used to extract the ROI[3]: 
1. Block direction estimation and direction variety check.

2. Morphological methods.

i) Block direction estimation

In order to estimate the block direction for each block of the fingerprint image with $\mathrm{WxW}$ in $\operatorname{size}(\mathrm{W}$ is 16 pixels by default), the following algorithm is used:

1. Calculate the gradient values along $\mathrm{x}$-direction $(\mathrm{gx})$ and $y$-direction (gy) for each pixel of the block. Two Sobel filters are used to fulfill the task. II. For each lock, use following formula to get the Least Square approximation of the block direction.

$\operatorname{tg} 2 \beta=2(\mathrm{gx} * \mathrm{gy}) /(\mathrm{gx} 2-\mathrm{gy} 2)$ for all the pixels in each block.

The formula is easy to understand by regarding gradient values along $\mathrm{x}$-direction and $\mathrm{y}$-direction as cosine value and sine value. So the tangent value of the block direction is estimated nearly the same as the way illustrated by the following formula[3].

$$
\operatorname{tg} 2 \theta=2 \sin \theta \cos \theta /(\cos 2 \theta-\sin 2 \theta)
$$

After the estimation of each block direction, the blocks without significant information on ridges and furrows are discarded based on the following formulas:

$$
\begin{gathered}
\mathrm{E}=\left\{2 \sum \sum(\mathrm{gx} * \mathrm{gy})+\sum \sum(\mathrm{gx} 2-\mathrm{gy} 2)\right\} / \\
\mathrm{W}^{*} \mathrm{~W}^{*} \sum \sum(\mathrm{gx} 2+\mathrm{gy} 2)
\end{gathered}
$$

Where $\mathrm{E}$ is the certainty level. For each block, if $\mathrm{E}$ is below a threshold, then the block is regarded as a background block.

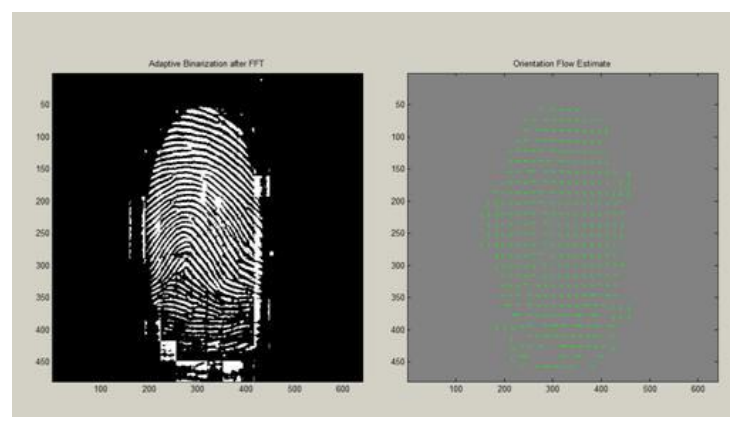

Fig10. Direction map. Binarized fingerprint (left), Direction map (right)

2. ROI extraction by Morphological operations The morphological operations adopted are:
a) OPEN
b) CLOSE

The „OPEN"e operation can expand images and remove peaks introduced by background noise [Fig 10]. The „CLOSE ${ }^{e c}$ operation can shrink images and eliminate small cavities [Fig $11]$.

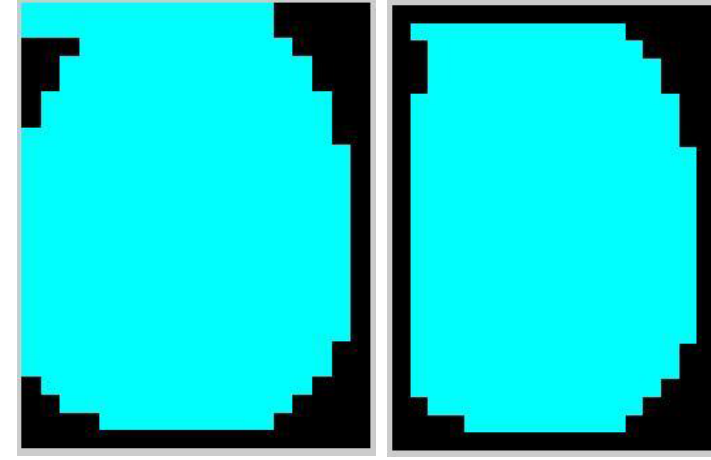

Fig 11. Original Image Area Fig 12. After CLOSE

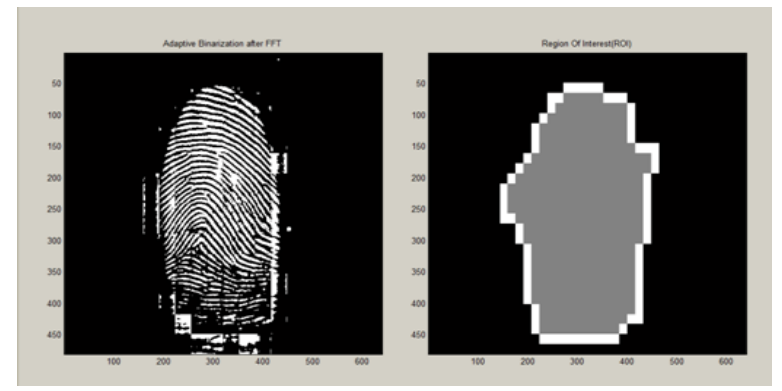

Fig 8. After OPEN operation Fig 9 . ROI + Bound

The bound is the subtraction of the closed area from the opened area. Then the algorithm throws away those leftmost, rightmost, uppermost and bottommost blocks out of the bound so as to get the tightly bounded region just containing the bound and inner area.

\section{MINUTIA EXTRACTION}

\subsubsection{Fingerprint ridge thinning:}

Thinning is used to eliminate the redundant pixels of ridges, till the ridges are just one pixel wide ridge. Iterative, parallel thinning algorithm is used for thinning. The algorithm marks down redundant pixels in each small image window $(3 \times 3)$ in each scan of the full fingerprint image. And finally removes all those marked pixels after several scans. To extract thinned ridges from gray-level fingerprint images directly, it uses a one-in-all method. Their method traces along the ridges having maximum gray intensity value. Since only pixels with maximum gray intensity value are remained, binarization is implicitly enforced[3].

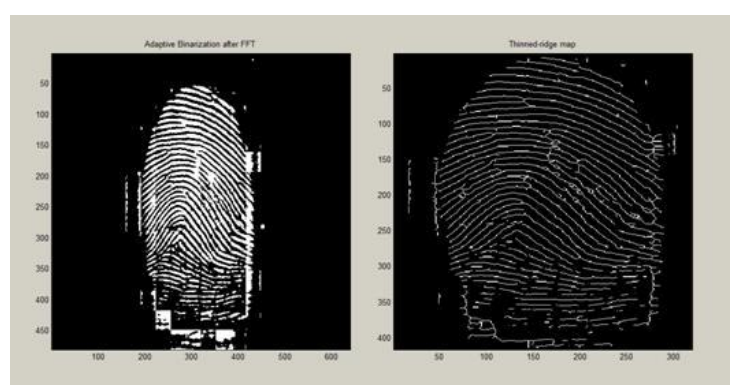

Fig.13. Thinning

\subsubsection{Minutia Match}

We consider two set of the minutia points and apply matching algorithm. An alignment-based match algorithm is used. It includes two consecutive stages: i) alignment stage and ii) match stage[3]. 
i. Alignment stage:

Given two fingerprint images to be matched i.e. composite image and image obtained dynamically,choose any one minutia from each image; calculate the similarity of the two ridges associated with he two referenced minutia points. If the similarity is larger than a threshold, transform each set of minutia to a new coordination system whose origin is at the referenced point and whose $\mathrm{x}$-axis is coincident with the direction of the referenced point. ii. Match stage: After we get two set of transformed minutia points, we use the elastic match algorithm tocount the matched minutia pairs by assuming two minutia having nearly the same position and direction are identical.

\subsubsection{Alignment Stage}

1. The ridge associated with each minutia is representedas a series of $\mathrm{x}$-coordinates $(\mathrm{x} 1, \mathrm{x} 2 \ldots \mathrm{xn})$ of the points on the ridge. A point is sampled per ridge length $\mathrm{L}$ starting from the minutia point, where the $\mathrm{L}$ is the average inter-ridge length. And $\mathrm{n}$ is set to 10 unless the total ridge length is less than $10 * \mathrm{~L}$.

So the similarity of correlating the two ridges is derived

$$
\mathrm{S}=\sum \mathrm{mi}_{\mathrm{i}}=0 \mathrm{Xi} \mathrm{Xi}_{\mathrm{i}} /\left[\sum \mathrm{mi}_{\mathrm{i}}=0 \mathrm{Xi} 2 \mathrm{X}_{\mathrm{i} 2}\right]^{\wedge} 0.5,
$$

where $(x i \sim x n)$ and $(\mathrm{Xi} \sim \mathrm{XN})$ are the set of minutia for each fingerprint image respectively. And $m$ is minimal one of the $n$ and $\mathrm{N}$ value. If the similarity score is larger than 0.8 , then go to step 2, otherwise continue to match the next pair of ridges.

2. For each fingerprint, translate and rotate all other

Minutia.

\subsubsection{Match Stage}

The matching algorithm for the aligned minutia patterns

needs to be elastic since the strict match requiring that all

parameters (x,y, $\square$ ) are the same for two identical minutia is impossible due to the slight deformations and inexact quantization's of minutia. A bounding box is placed around each template minutia. If the minutia to be matched is within the rectangle box and the direction discrepancy between them is very small, then the two minutia are regarded as a matched minutia pair. Each minutia in the template image either has no matched minutia or has only one corresponding minutia[3].

The final match ratio for two fingerprints is the number of total matched pair over the number of minutia of the template fingerprint. The score is $100 *$ ratio and ranges from 0 to 100 . If the score is larger than a pre-specified threshold, the two fingerprints are from the same finger. However, the elastic match algorithm has large computation complexity and is vulnerable to false minutia

\section{SIGNATURE 4.2 Types of Signature}

There are two different approaches of signature verification.

\subsection{Off-Line or Static Signature Technique:}

This approach is based on static characteristics of the signature which are invariant. The signature recognition here becomes a typical pattern recognition task knowing that variations in signature pattern are inevitable; the task of signature authentication can be narrowed to drawing the threshold of the range of genuine variation. The images of the signatures written on a paper are obtained using a scanner or a camera in offline systems.
1.2. On-line or Dynamic Signature Technique:

The second type of signature verification technique is online or dynamic system. This technique is based on dynamic characteristics of the process of signing. This verification uses signatures that are captured by pressure sensitive tablets that extract dynamic properties of a signature in addition to its shape.The number of order of the strokes, the overall speed of the signature and the pen pressure at each point etc are the dynamic features that make the signature more unique and more difficult to forge. Application areas of Online

Signature Verification include protection of small personal devices (e.g. PDA, laptop), authorization of computer users for accessing sensitive data or programs and authentication of individuals for access to physical devices or buildings.

\subsection{Preprocessing}

The design of any offline signature verification system generally requires the solution of five sub problems: data acquisition, pre-processing, feature extraction, comparison process and performance evolution. The main aim to perform Pre-processing on an image is to enhance the image quality and obtain a transformed image.

The steps involved in Preprocessing are:-

a) Conversion

b) Noise Removal

c) Rotation

d) Smoothing

e) Thinning

f) Signature Extraction

g) Normalization

a) Converting colored image to gray scale images

We get the output of the scanning devices and image

Capturing devices in color format.. A coordinate matrix and three color matrices constitutes the colored image. The Coordinate matrix contains X, Y coordinate values of the image.

Gray color $=0.299 *$ Red $+0.5876 *$ Green $+0.114 *$ Blue

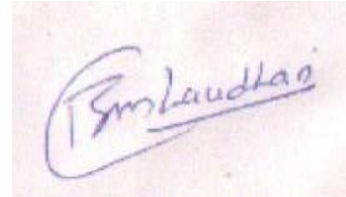

Fig.14 original image

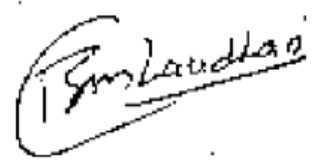

fig.15 gray scale image b) Noise removal: The noise removal involves removal of the unwanted pixels from the image. Noise reduction is also "smoothing" or "noise filtering". Images are often damaged because of positive and negative impulses stemming from decoding errors or noisy channels. Undesirable effects due illumination and other objects in the environment may also degrade a image. Median filter is widely used for smoothing and restoring images corrupted by noise. For reducing impulsive or salt-and-pepper type noise ,median filter is used which is a nonlinear process. In a median filter, a window slidesover the image, and for each positioning of the window, the median intensity of the pixels inside it determines the intensity of the pixel located in the middle of the window.[6] 


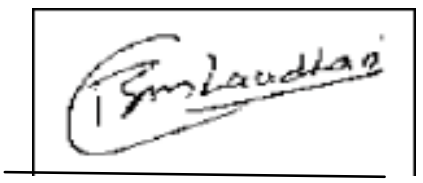

Fig.16 noise removal

c) Rotation: Rotation of a signature is necessary as time domain approaches are sensitive to angle variations compared to frequency domain approaches. It coincides the axis of inertia of all the signatures to the same horizontal axis. The signature is then rotated clockwise to remove skewness.

d) Smoothing: Smoothing is performed to remove noice from signature and to expose its feature for further featuring . The adaptive filter, which preserves edges and high frequency components of the signature, is used for smoothing.

e) Thining: Thining is a morphological process necessary for the reduction of data and time. . It consists of two subiterations: one aimed at deleting the south-east boundary points and the northwest corner points while the other one is aimed at deleting the north-west boundary points and the southeast corner points. It reduces the signature to a skeleton of unitary thickness.

f) Signature extraction : The extra background created due to rotation is removed by extracting the smallest box that covers the signature. The smallest box is determined by the height and width of the signature and is then cropped to the measured dimension. For this purpose, thresholding is used widely. This can be done by choosing the threshold value $\mathrm{H}$. the pixel values that are less than or equal to $\mathrm{H}$ are assigned to 0 and those greater than $\mathrm{H}$ to 1.This is done to separate the signature pixels from the background pixels. Clearly we are interested in dark objects on a light background, and so the brightness threshold, is approximately chosen and that threshold value is applied to image pixels $f(x, y)$ as in the equation

(2)[6]

If $\mathrm{f}(\mathrm{x}, \mathrm{y}) \geq \mathrm{H}$ then

$\mathrm{f}(\mathrm{x}, \mathrm{y})=$ Background

else $\mathrm{f}(\mathrm{x}, \mathrm{y})=$ Object

The signature image obtained by separating it from the

complex background detail is converted into binary image white background taking the pixel value of 1 .

g) Normalisation :

Normalization is required to standardize the size of signatures having interpersonal and intrapersonal differences. Due to the irregularities in the image scanning and capturing process, the image dimensions may vary. Also, height and width of signatures vary from

person to person and, sometimes, even the same person may use different size signatures. For that we need to remove or minimise the size differences and obtain a standard signature size for all signatures. All signatures will have the same dimensions after this normalisation process.. During the normalization process, the aspect ratio between width and height of a signature is kept intact.[6] Normalization process is done by using the following

eq.(3) \& (4)

$$
\mathrm{Xi}=\frac{\mathrm{Xi}_{\mathrm{i} 1-\mathrm{X} \min \mathrm{XM}}}{\mathrm{Xmax}_{\mathrm{m}} \mathrm{X} \min }
$$

$\mathrm{yi}=\underline{\mathrm{yi} 1-\mathrm{ymin} \mathrm{x} M}$.

ymax-ymin

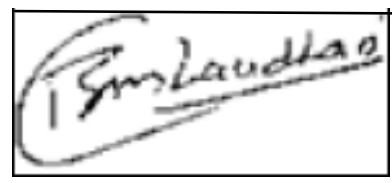

Fig.17 normalized image

\section{h) Gray Scale Image}

Images that are without color, or achromatic images are grayscale images. The levels of a grayscale range from 0 (black) to 1 (white).

\section{FEATURE EXTRACTION}

To improve the accuracy of the signature verification system we perform the task of feature extraction. The following are the features that are extracted from the pre-processed image[4]:

a) Height to width ratio (F1):

The ratio of height to width of the signature is the feature F1. The coordinates of the bounding box of the cropped signature is determined. By using these coordinates the height and the width are computed. At different times the

height and width can change for a person. But the ratio of height and weight of an individuales signature is approximately constant.

$\mathrm{F} 1=$ ( height of the signature/ width of the signature $)$

b) Occupancy Ratio (F2):

It is the ratio of number of pixels which belongs to the signature and the total number of pixels of the signature

image. The information about the signature density is provided by this feature. F2= (number of pixels which belongs to the signature/ total number of pixels in the Signature image)

c) Density ratio (F3): The signature image is divided into two halves vertically. F3 is the ratio of number of pixels which belong to the left half of the signature image to the number of pixels which belong to the right half of the signature image. The signature density ratio of the two

halves of the signature image information is provided by

F3.

$\mathrm{F} 3=$ (density of the left half of the signature/density of the right half of the signature)

d) Critical points (F4): The large variation in the intensity in all the directions is found in the regions of corners. The corner 
points are treated as the critical points. These critical points are counted in the signature image. The counting is done by using Harris corner method.

e) Center of gravity (F5): the number of the white pixels in the binary image is treated as ON pixel. F5 is the average coordinate point of all $\mathrm{ON}$ pixels of the binary signature image.

f) Slope of center of gravity (F6)- The signature image is divided into two halves. The center of gravity of each part is determined separately. The slope of the line joining the center of gravities is determined.

g) Center of masses of sub-regions (F7)- Firstly the signature is divided vertically to get two center of masses. Then, each half of signature images is divided horizontally to get four center of masses. Again, four regions of signature image are divided vertically to get eight centers of masses. Finally, eight regions of signature image are divided horizontally to get sixteen

centers of masses. The feature F7 is the above thirty

centers of masses of the signature image.

The above features F1 to F7 are extracted and stored in a feature vector. This feature vector is used to train the system as well as for verification of a sample signature.

4 Algorithm for signature verification using Euclidean Distance[5]:

i. Input the set of signatures of a person.

ii. Convert the image into gray scale and binary image

iii. Perform noise reduction on both images

iv. Perform the rotation on binary image in order to

equalize the inclination based on the baseline slant.

v. Find the bounding box of image and crop the image

vi. Extract the features F1, F2, F3, F4, F5, F6 and F7 from the signature and store in a feature matrix.

vii. Dataset is created by computing the mean signature feature values.

viii. Calculate the Euclidean distance of the query signature features from mean signature features of the dataset.

ix. If the distance is below a certain threshold then query signature is verified to that of the claimed person otherwise it is detected as a forged one.

\section{EXPERIMENTAL RESULTS}

\subsection{Results and Discussions}

\subsection{Experimental Results for fingerprint recognitions:}

We have two different techniques to obtained a composite minutiae set .The two minutiae sets are -

1] Obtained by extracting from the composite image (MR1)

2] Obtained by integrating individual minutiae sets (MR2)

These sets are treated as template minutiae sets against which the query minutiae set can be matched. The four different impressions of the same finger where obtained. The first two impressions were used to construct the composite minutiae set of a finger. The other two impressions where used as query images. In this way ,for performing the mosaicking algorithm the four impressions of each of 8 subjects where obtained. The composite image for each user was generated. The average number of minutiae increases from 36 to 45 after mosaicking. Matching was accompanied using minutiae matching algorithm. The matching performance is observed to improve significantly when the composite template is used instead of individual impressions therefore this gives best performance. Consider a query image IU with the minutaie set MU and the template minutiae sets MP, MQ, MR1 , MR2 ,

so the following comparisons are made-

1) MU with MP

2) Mu with $M_{Q}$

3) Mu with MR1

4) Mu with $M_{R 2}$

The ROC curves depicts the performance of these 4 different matchings. From the graph it is cleared that composite images results in improved matching performance. Better matching performance is given by MR1, rather than MR2, which may be due to the incorrect minutaie orientation in MR2.

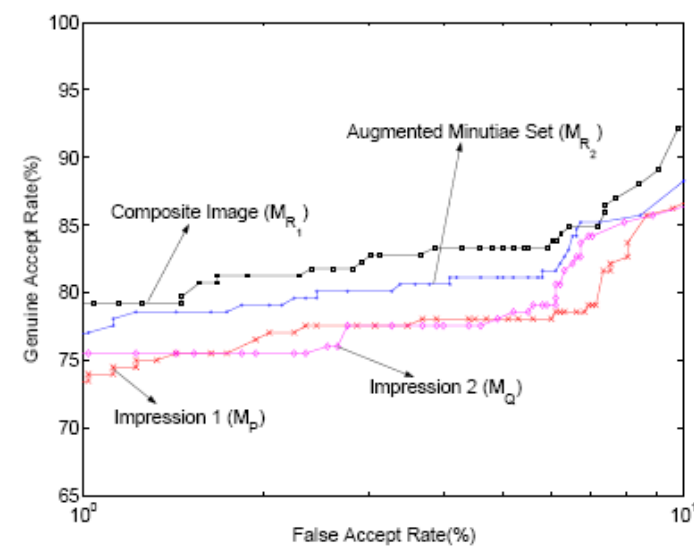

Fig.18 The ROC curves indicating improvement in matching performance after mosaicking templates.

\section{CONCLUSION AND FUTURE WORK}

For fingerprint we have describe the construction technique for the composite template that integrates the information available in two different impressions of same finger. In order to established an initial approximate alignment the corresponding minutiae points are used. For registering the two impressions a modified ICP algorithm is used. These ICP algorithms generate the transformation matrix and construct the composite template. The minutiae points are extracted from this composite template and are tested against the query image of the same finger. For the signature the proposed method promises simple and reliable solutions to the problem of signature verification It includes an image clustering process based on Euclidian distance approach . This approach enables the handling of clusters of different sizes and shapes of signatures. Hence in this way the fingerprint recognition and signature verification takes place thereby giving satisfactory accuracy.

Both this concepts can be used together in the construction of stylus providing a high security and authentication of user. The construction of stylus is such that is captures the fingerprint images as well as matches the user signature. This pen can be further use in many government as well as private companies to prevent unauthorized access to the system. 


\section{REFERENCES}

[1] A. Jain and A. Ross. Fingerprint mosaicking. In Proc. of IEEE International Conference on Acoustics, Speech, and Signal Processing (ICASSP), volume 4, pages 4064\{4067, Orlando, FL, May 2002.

[2] FINGERPRINT MOSAICKING, Anil Jain and Arun Ross Michigan State University, East Lansing, MI, USA 48824,fjain, rossarung@cse.msu.edu, IEEE International Conference on Acoustics, Speech, and Signal Processing (ICASSP), Orlando, Florida, May 13 - 17, 2002.

[3] wuzhilli(Vincent AT comp.hkbu.ed.u.hk) OEW 801, Hong Kong Baptist University.

[4] Ranjan Jana et al, / (IJCSIT) International Journal of Computer Science and Information Technologies, Vol. 5 (1) , 2014, 707-710 Offline Signature Verification using Euclidian Distance.
[5] Berthold K. P. Horn, "Closed-form solution of absoluteorientation using unit quaternions," Journal of the Optical Society of America, vol. 4, no. 4, pp. 629642, April 1987.

[6] International Journal of IT, Engineering and Applied SciencesResearch (IJIEASR) Volume 2, No. 1, January 2013 SignatureRecognition \& Verification System Using Back PropagationNeural Network.

[7] Md. Maruf Monwar and Marina Gavrilova "FES: A System for Combining Face, Ear and Signature Biometrics using Rank Level Fusion”. Fifth International Conference on Information Technology: New Generations. 